\title{
MMW-HOTLINE
}

Leser der MMW können sich mit allen Fragen zur Abrechnung und Praxisführung an Helmut Walbert, Facharzt für Allgemeinmedizin, Würzburg, wenden. Sie erreichen ihn jeden Donnerstag von 13 bis 15 Uhr unter der kostenlosen Rufnummer (0800) 2379830 oder per E-Mail: w@lbert.info.

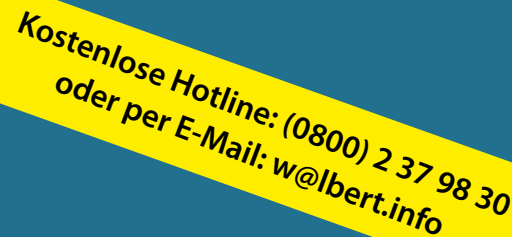

Helmut Walbert

Allgemeinarzt, Medizinjournalist und Betriebswirt Medizin

IGeL

\section{Welche Sachkosten gibt es da extra?}

Dr. med. J. Sch., Allgemeinarzt, Veitshöchheim:

Mein Praxispartner hat bei einer Individuellen Gesundheitsleistung (IGeL) Sachkosten für Spritzen und Kanülen abgerechnet. Der betreffende Patient weigert sich: Diese Kosten habe er bisher nicht bezahlen müssen. Was ist korrekt?

Antwort: Auch IGeL unterliegen den Vorschriften der GOÄ. Die Erstattung von Sachkosten ist in $\S 10$ der GOÄ „Ersatz von Auslagen" geregelt. Hier ist im Absatz (1) festgehalten: „Neben den für die einzelnen ärzt- lichen Leistungen vorgesehenen Gebühren können als Auslagen nur berechnet werden (1) die Kosten für diejenigen Arzneimittel, Verbandmittel und sonstigen Materialien, die der Patient zur weiteren Verwendung behält oder die mit einer einmaligen Anwendung verbraucht sind, soweit in Absatz 2 nichts anderes bestimmt ist."

Der Absatz 2 zählt dann die ausgeschlossenen Kosten auf: „(2) Nicht berechnet werden können die Kosten für Kleinmaterialien wie Zellstoff, Mulltupfer, Schnellverbandmaterial, Verbandspray, Gewebekleb- stoff auf Histoacrylbasis, Mullkompressen, Holzspatel, Holzstäbchen, Wattestäbchen, Gummifingerlinge, sowie für (5) folgende Einmalartikel: Einmalspritzen, Einmalkanülen, Einmalhandschuhe, Einmalharnblasenkatheter, Einmalskalpelle ..... Damit ist der Sachverhalt eindeutig: Der Patient muss nicht zahlen. Grundsätzlich hat der Kollege aber Recht: In vielen Arztpraxen werden die Sachkosten nämlich gar nicht verrechnet. Je nach Leistungsstruktur der Praxis führt dies zu einem durchaus spürbaren Honorar- bzw. Gewinnverlust.

\section{Fotokopien von Befunden und Arztbriefen}

\section{Kann man die irgendwie verrechnen?}

Frau M., Medizinische Fachangestellte in Gemeinschaftspraxis, München:

Wir machen nahezu täglich Kopien von Befunden, Arztbriefen usw. Sind diese Kopien irgendwie verrechenbar?

Antwort: Grundsätzlich sind Kopien für den Eigenbedarf der Praxis nicht verrechenbar. Eine Ausnahme sind Formulargutachten für Versicherungen: Hier kann eine Kopie des Gutachtens für die Akten in der Praxis dem Auftraggeber in Rechnung gestellt werden mit der GOP 96, je Kopie $€ 0,17$. Dies wird von den Versicherungen auch anstandslos akzeptiert, wenn es sich um individuelle versicherungsspezifische Formulare handelt. Ansonsten ist die GOP 96 "nur neben den Leistungen nach den Nummern 80, 85 und
90 und nur mit dem einfachen Gebührensatz berechnungsfähig".

Häufig wollen Patienten Kopien von Arztbriefen, Labor- und sonstigen Befunden. Dies muss den Patienten privat verrechnet werden. Hier sollten mindestens die ortsüblichen Kopierkosten verlangt werden. Dies hat auch eine erzieherische Wirkung in Richtung Kostenbewußtsein: „Wenn es mich nichts kostet, kann ich meinem Sammeltrieb uneingeschränkt nachgehen".

Im Rahmen des EBM, also bei der GKV, gibt es die GOP 40 144, „Kostenpauschale für fotokopierte oder EDV-technisch reproduzierte Befundmitteilungen, Berichte, Arztbriefe und andere patientenbezogene Unterlagen ausschließlich für den mit- oder weiterbehandelnden oder konsiliarisch tätigen Arzt oder den Arzt des Krankenhauses, je Seite $€ 0,13$.. Diese GOP gilt nur für Kopien im Rahmen der vertragsärztlichen Versorgung. Fotokopien für den Medizinischen Dienst, Betriebsärzte, Ärzte der Unfall- oder Rentenversicherung o. ä. fallen nicht hierunter. Können diese den jeweiligen Institutionen nicht in Rechnung gestellt werden, müssen sie nach GOÄ dem Patienten abverlangt werden.

Es lohnt sich, bei nächster Gelegenheit die Anzahl der Kopien mal zu erfassen. In vielen Praxen kommen 500 Kopien im Monat zusammen. Nach GOP 40144 sind dies immerhin $65 €$ im Monat, bzw. $780 €$ im Jahr! 